Usages du français et pratiques d'enseignement en Europe balkanique, centrale et orientale - Grèce,

Serbie, Bulgarie, Moldavie, Hongrie, Allemagne, Russie - XVIIIe - XXe siècles

\title{
La Mission laïque de Salonique : berceau et vecteur de la francophonie en Europe du sud-est de 1906 à la fin des années 1940
}

Lampros Flitouris

\section{(2) OpenEdition}

\section{Édition électronique}

URL : https://journals.openedition.org/dhfles/4194

DOI : $10.4000 /$ dhfles.4194

ISSN : 2221-4038

Éditeur

Société Internationale pour l'Histoire du Français Langue Étrangère ou Seconde

Édition imprimée

Date de publication : 1 juin 2015

Pagination : 97-115

ISSN : 0992-7654

\section{Référence électronique}

Lampros Flitouris, «La Mission laïque de Salonique : berceau et vecteur de la francophonie en Europe du sud-est de 1906 à la fin des années 1940 », Documents pour l'histoire du français langue étrangère ou seconde [En ligne], 54 | 2015, mis en ligne le 19 novembre 2017, consulté le 29 mars 2023. URL : http:// journals.openedition.org/dhfles/4194; DOI : https://doi.org/10.4000/dhfles.4194

Ce document a été généré automatiquement le 29 mars 2023.

Tous droits réservés 


\title{
La Mission laïque de Salonique : berceau et vecteur de la francophonie en Europe du sud-est de 1906 à la fin des années 1940
}

\author{
Lampros Flitouris
}

\section{Introduction}

1 Salonique, la capitale du pachalik de Macédoine et la deuxième ville la plus peuplée de l'Empire ottoman, est un exemple particulier de l'influence qu'avaient la langue et la culture françaises dans l'Europe du sud-est. Centre commercial et économique, porte ouvrant sur les Balkans avec une population multinationale très active, Salonique a attiré l'intérêt des Européens grâce aux atouts dont elle disposait, notamment en matière d'activités financières. Pour cette raison, plusieurs maisons de commerce étrangères ont développé (individuellement ou en collaboration avec des commerçants locaux grecs, juifs ou arméniens) une activité économique importante notamment à partir du XVIII ${ }^{\mathrm{e}}$ siècle. Les Français, pionniers de la collaboration avec les Ottomans grâce à la tradition des capitulations, ont installé un réseau de consulats et de représentants commerciaux qui a permis au capital français d'avoir un rôle de premier rang dans la vie économique de l'Empire (Thobie 1977 : 13-45). Les Français se sont installés à Salonique à la fin du XVII ${ }^{e}$ siècle et notamment au début du XVIII ${ }^{\mathrm{e}}$ siècle, en organisant une première communauté qui bénéficiait d'une série de droits sous la protection du consulat français de la ville. Du moment où les interlocuteurs des Français étaient les Grecs, les juifs et les Arméniens de la ville, la langue française est devenue l'arme linguistique indispensable sur les marchés. Les commerçants de Salonique ont compris assez tôt que l'apprentissage du français pourrait faciliter les affaires, ce qui explique l'ouverture des écoles françaises dans la ville (Pantelodimos $1985: 146-155)$. 
2 Dans la ville, il existait déjà une tradition francophone grâce aux écoles catholiques et à celles de l'Alliance israélite universelle. En outre, de nombreuses écoles européennes s'adressaient non seulement aux communautés respectives (française, anglaise, italienne, allemande mais aussi roumaine, bulgare ou serbe) mais notamment aux juifs et aux Grecs qui appartenaient aux classes économiquement puissantes (Kontogianni 1910: 176-178). La diffusion des langues européennes a contribué à diffuser la propagande des puissances sur la société locale et, bien évidemment, au développement des intérêts des pays européens et balkaniques pendant une époque décisive pour l'avenir de Salonique. Contrairement aux écoles des minorités ethniques de la ville (turques, bulgares, roumaines et serbes) qui fermeront progressivement à partir de 1913 et jusqu'à la fin des années 1920, les écoles des juifs et des Arméniens ont poursuivi leurs cours dans le cadre de l'État grec. Les écoles allemandes et italiennes ont travaillé normalement pendant l'entre-deux-guerres et elles ont même participé à la guerre des propagandes de l'entre-deux-guerres (Enepekides 1982: 118-119; Tsirpanlis $1987: 120$ ).

3 Dès avant 1923, le français jouait le rôle de langue commune (avec le grec), utilisé pour la communication non seulement pour les affaires dans les bazars de la ville mais, parfois même, pour la communication intra-communautaire. Après l'arrivée des immigrés grecs de l'Asie Mineure, l'influence du français dans le commerce se réduit progressivement. En même temps, les services publics, comme la poste ou les chemins de fer, continuaient à utiliser le français comme langue de communication parallèlement au grec. Pour la communauté juive qui, après 1923, est devenue la deuxième plus grande communauté de la ville derrière les Grecs, le français demeura pendant cette époque la langue de l'enseignement et celle des journaux et du commerce (Moutsopoulos 1992: 22-32). Le français était aussi la langue de l'intelligentsia salonicienne; il était utilisé dans les théâtres et les conférences artistiques où le français fut la lingua franca d'un public multiethnique. La musique française était la musique favorite de l'orchestre municipal de la ville dès sa formation en 1886. Même dans les deux loges maçonniques de la ville, dont les membres appartenaient aux sections grecques du Grand Orient de France, l'usage du français était dominant. La majorité des autochtones étant ainsi habituée à cette langue, l'usage public du français semblait normal.

4 La présence de l'Armée d'Orient pendant la Grande Guerre dans la région a renforcé l'utilisation de la langue française notamment par les habitants qui ont travaillé avec les services de l'armée alliée (Vafopoulos 1985 : 28). En outre, l'œuvre pacifique de l'Armée d'Orient, avec ses fouilles et recherches archéologiques, son aide sanitaire ou la construction d'œuvres d'intérêt public (par exemple des rues ou des ponts), a gagné la sympathie des habitants. Ce souvenir positif resta vivace pendant des années dans la mémoire collective du peuple de la région (Hatzopoulos \& Hautefeuille 1992: 191-200 ; Skourtis 1992: 201-205). Les écoles catholiques et les grandes institutions d'enseignement laïque, les journaux francophones, les hôpitaux français et les ligues franco-helléniques ont relayé la présence culturelle française en Grèce et ont donné à la francophonie une place particulière que le lycée de la Mission laïque de Salonique a renforcée. 


\section{La Mission laïque de Salonique de sa fondation aux années 1930}

5 L'organisation de la Mission laïque française, fondée à Paris en 1902 par un groupe d'intellectuels, est reconnue par l'État en 1907 comme une des institutions chargées de la diffusion de la langue et de la culture françaises. En appliquant une politique d'enseignement plus progressiste que les autres fondations similaires, son but était la diffusion de la culture française en respectant, toutefois, les cultures des pays d'accueil. Son principal lieu d'activité était la Méditerranée orientale et la première école s'établit à Salonique en 1906, initiative qui soulignait l'importance de la ville au carrefour de l'Europe et de l'Asie pendant une période d'affrontements et de rivalités dans la région. Dans les années suivantes la Mlf a inauguré d'autres écoles en Méditerranée orientale (Beyrouth 1909, Le Caire 1910, Damas 1923 etc.).

6 L'école de la Mission fut la première grande institution d'enseignement laïque de langue française qui a développé une activité scolaire à Salonique. La Mission y a organisé un lycée de garçons, un lycée de filles, une école primaire et une école de commerce. Ses établissements s'installeront dans un grand complexe de bâtiments où les élèves avaient la possibilité de recevoir une éducation originale. En 1910, elle accueillait 290 garçons, 100 filles et 22 étudiants à l'école de commerce de la Mission (Moschou 1994 : 267-275). À partir de 1912, les diplômes délivrés étaient équivalents à ceux des écoles grecques et ils étaient reconnus par les autorités helléniques comme à l'époque de la domination ottomane. Au début de la période d'administration grecque, cette reconnaissance était basée sur le droit coutumier et non sur un accord entre l'État grec et les Français. L'acceptation par les Grecs du fonctionnement de la Mission dans le même cadre qu'avant 1912 n'était qu'une expression de sympathie et de confiance des Grecs pour le rôle de cet établissement (Skourtis 1986 : 342). En vérité, l'administration hellénique était mal organisée. La période qui suit les guerres balkaniques et la suite des événements ont permis aux Grecs de s'occuper plus sérieusement des écoles étrangères de Salonique seulement après 1923. L'école de la Mission bénéficiait de franchises mais en même temps les professeurs payaient des taxes. Par contre, les aides financières privées ou celles de l'État français destinées à l'amélioration des œuvres scolaires, étaient libres de taxes. Les services d'éducation du pays reconnaissaient leur droit de faire des contrôles dans l'école, notamment dans le domaine de l'hygiène, mais, dans les faits, ce contrôle était très symbolique (AYE, 14/1/1914). Le programme des cours était le même que celui des lycées français et les professeurs français étaient détachés auprès du ministère des Affaires étrangères français par le ministère de l'Instruction publique. L'État grec avait réussi à imposer l'enseignement de la langue grecque, de l'histoire et de la géographie du pays. L'enseignement des cultes qui était incorporé au programme de l'école n'était pas obligatoire pour les juifs et les catholiques. Pendant les examens à la fin de l'année, la présence des inspecteurs grecs envoyés par le ministère était possible et ces fonctionnaires avaient le droit de poser aux élèves des questions durant l'examen (AYE, 16/6/1914). En réalité, l'histoire et la géographie n'étaient pas enseignées. Ce fait a provoqué les réclamations du ministère hellénique à partir de 1914. Les responsables grecs soulignaient le danger d'une éducation grecque incomplète pour les élèves de nationalité hellénique et ils demandaient le droit de contrôle non seulement sur la partie grécophone du programme mais sur la totalité des cours. Les demandes grecques touchaient aux droits 
français établis depuis des années et s'opposaient à une mentalité dominante qui avait ses racines à l'époque des capitulations ottomanes. Or, pour l'État grec, ce changement $\mathrm{du}$ cadre dans lequel fonctionnaient les écoles étrangères, était nécessaire pour incorporer avec succès les populations des provinces nouvelles dans le système hellénique et pour montrer l'autorité des institutions et des services de la nouvelle administration.

7 À partir de 1914, Jules Lecoq fut le directeur du Lycée. La première étape de sa direction (1914-1918) a coïncidé avec la présence des troupes alliées à Salonique pendant la Grande Guerre. Durant cette période, les établissements de la Mission ont accueilli des officiers français. La Mission est devenue le centre de la propagande pro-alliée en Macédoine et elle publiait un journal propagandiste intitulé Tricolore. Au Lycée fut également assurée la distribution de la Revue macédonienne, publiée par l'Armée d'Orient. Pendant cette période, Lecoq travaillait plutôt comme un agent politique de la République que comme un enseignant. Certaines de ses idées ont provoqué la suspicion des Grecs en particulier celles concernant l'internationalisation de Salonique et la formation d'un état macédonien avec des cantons ethniques. L'attitude de Lecoq a provoqué la colère des Grecs et à partir de cette époque le rôle de la Mission devint très suspect aux yeux des habitants orthodoxes, alors que nombre de Français étaient sensibles à ces idées présentées par les juifs de la ville.

Pendant les années vingt et trente, les activités de la Mission se sont poursuivies dans un climat antisémite dominant. Malgré le changement de direction en 1919 - Ozou remplace Lecoq - les suspicions grecques continuaient d'exister comme auparavant (Revue de l'enseignement du français hors de la France 1926: 268-269). Chaque fois que la tension montait entre les juifs et les orthodoxes, la Mission recevait des attaques des deux parties et sa position devenait très délicate. Les juifs l'accusaient de ne pas respecter le fait que la majorité de ses élèves était juive. Les orthodoxes l'accusaient de pratiquer une propagande pro-sioniste ou de faciliter une telle activité. En 1921, l'intempérance de langue d'un jeune professeur français au Lycée provoqua un incident entre la Mission et la communauté israélite. Pendant cette période les sionistes de la ville, soutenus par le journal Pro-Israël, trouvaient que la Mission facilitait l'assimilation des juifs à la société hellénique. En transmettant dans la ville de Salonique la différence qui existait à Paris entre l'Alliance israélite universelle et les sionistes, on accusait la Mission de discriminations raciales et d'utiliser des méthodes tyranniques à l'égard des élèves juifs (Dumont 1995 : 131-150 ; ADN, 3/4/1922). En 1929, la Mission fut accusée de nouveau par les sionistes de la ville de racisme après un nouvel incident entre un professeur grec et un élève juif. (Makedonia 20/10/1929; Le Progrès 23/10/1929; L'Indépendant 23/10/1929). De son côté, la presse grécophone et notamment le journal Makedonia accusait la Mission de jouer un rôle pro-juif (Tousimis $1990: 110-120$ ). Cette tension a inquiété la direction générale de la Mission à Paris et pour éclairer l'affaire, le conseil d'administration a envoyé en mission le secrétaire général et le trésorier de l'association à l'automne 1929. Les responsables français ont conclu que le problème avait deux causes : a) les problèmes interethniques de la société de la ville et b) le grand nombre d'élèves qui était obligé de partager des locaux insuffisants. Malgré tout, le lycée était obligé de poursuivre sa politique et son activité " dans le sens de l'union, du rapprochement entre tous les éléments de la population » (Revue de l'enseignement $d u$ français hors de la France 1930: 71-74). En 1931, après le pogrom antijuif de Kambel, 
l'armée hellénique fut obligée de placer sous sa protection l'école française de peur d'une attaque par des extrémistes de droite.

9 Un autre aspect de l'activité de la Mission à la fin des années vingt concerne les relations développées avec les Serbes. Sous le prétexte que dans la province de Serbie du sud (actuelle Ancienne République Yougoslave de Macédoine), n'existait qu'une école franco-catholique à Skopje, la Mission a organisé une classe pour des élèves serbes. Un professeur serbe, payé par Belgrade, donnait des cours de serbe aux élèves qui provenaient des familles riches de Serbie. En 1928, la Mission hébergeait 30 Serbes et un professeur serbe. La presse de Salonique publia une série de pamphlets contre "cette propagande serbe indirecte». Cette attaque de la presse était, peut-être, un signe de la tension existant dans les relations politiques gréco-serbes à cause du sujet de la zone de libre-échange, accordée aux Serbes dans le port de Salonique (GrumelJacquignon 2002: 53-56). Les problèmes sont devenus plus graves au moment où la direction de la Mission a décidé que l'enseignement du grec ne serait pas obligatoire pour les Serbes. Les autorités grecques rappelaient aux diplomates d'Athènes que la Mission utilisait comme professeurs de grec des diplômés des lycées helléniques et non des diplômés des universités. De plus, elles accusaient la direction de l'établissement de préférer pour les postes de l'administration plutôt les Arméniens et les juifs que les Grecs (AYE 8/12/1928). Pendant les années trente et après les fortes protestations de l'administration grecque, la Mission dut arrêter les cours de serbe, et par la suite, elle perdit cette clientèle.

10 Il faut souligner que la période entre la mort d'Ozou, en 1926, et l'arrivée de Havard comme directeur, en 1935, se caractérise par de graves problèmes administratifs. Le Lycée a connu trois directions différentes dans une période de neuf ans (Vattier 1926-1929, Chardron 1929-1930, Roulleau 1930-1935), ce qui posait des problèmes. Le directeur devait être capable de modération dans une société où les différences ethniques, religieuses et économiques avaient des répercussions sur la présence culturelle française. Dans les années vingt les inscriptions à la Mission augmentent en nombre (1 000 élèves en 1920, 1300 élèves en 1927). Pourtant, au début des années trente, à cause de la loi Papandréou qui interdisait l'inscription des élèves de moins de 11 ans dans les écoles étrangères, le nombre des élèves a diminué (519 en 1931 et 459 en 1932).

\section{La Mission laïque de Salonique des années trente à l'aventure de la Seconde Guerre mondiale}

11 Une période particulière de l'histoire du Lycée fut celle de 1935 à 1940 durant laquelle au poste de directeur se trouvait André Havard. Cet instituteur français a essayé d'abord de changer l'image que la société locale avait de son établissement. Animé peut-être par ses opinions personnelles, Havard n'était pas satisfait du fait que le succès de l'école concernait surtout les juifs de la ville. D'après lui, si l'école continuait à accueillir majoritairement des juifs pendant une période où l'antisémitisme progressait dans la ville, les relations des Français avec les Grecs ne pourraient pas s'améliorer. Cette situation menaçait la totalité de l'activité culturelle de la France à Thessalonique. Havard croyait que la diminution du nombre des élèves entre 1930 et 1935 était le résultat du climat qui existait mais surtout de l'ingratitude des juifs qui, parfois, préféraient s'inscrire dans les écoles italiennes et allemandes ou bien immigrer vers la 
France. Ces explications de Havard semblent peu justifiées. Il ne se rendait compte ni du changement de politique de l'État grec face au rôle des écoles étrangères à partir de 1931 ni du refus des Allemands d'accepter des élèves juifs dès 1933. Par contre, il essayait dans son rapport d'activité de la période 1935-1940 adressé aux autorités de Vichy, de présenter ses efforts pour l'amélioration de la situation, comme gigantesques et il décrivait l'activité de la période en utilisant des arguments très négatifs pour les orthodoxes et pour les juifs de la ville. Dans le rapport de septembre 1940 on peut lire sur l'avenir des rapports de l'école avec la communauté juive :

Il fallait de toute évidence, désolidariser le Lycée de l'élément israélite, pour échapper aux fluctuations de sa population et aux variations de son goût. Il fallait élargir le recrutement et l'étendre, après tant d'attente perdue, à l'élément grec de la ville de Salonique, et même au-delà si possible. (AMAE, 9/1940)

Havard déclarait avec emphase qu'avant son arrivée à Salonique, toute la vie culturelle était sous le contrôle des juifs :

Il n'est nullement exagéré d'affirmer que l'élément français n'avait pas de relations avec l'élite grecque de la ville. Le Lycée était considéré comme un établissement juif. Il importait donc de mettre un terme à cet état de choses qui durait depuis la fondation du Lycée et supprimer le monopole israélite de l'influence intellectuelle française, afin de pénétrer plus aisément et directement dans les milieux grecs orthodoxes. Je savais que cette œuvre était dangereuse et que les forces occultes juives omnipotentes réagiraient vigoureusement. Mon renvoi fut demandé à plusieurs reprises, mais mes supérieurs hiérarchiques de Paris ne jugèrent pas bon d'interrompe mes efforts et de compromettre les résultats acquis. Ces attaques ne réussirent qu'à me créer des difficultés jusque dans l'établissement dont j'assumais la direction. (Ibid.)

13 Face aux arguments avancés par le directeur du lycée, Havard essaye de justifier son activité comme le résultat de la pression de la société locale. Il est vrai que pendant les années trente le nombre des élèves juifs a diminué en raison de la diminution générale de la population juive de la ville, et aussi que selon les habitants, la Mission était liée avec les juifs à cause de la francophilie traditionnelle de cette communauté. À cause de la politique de Havard, le nombre des élèves juifs à baissé de $74 \%$ à $47 \%$ et le nombre des élèves orthodoxes est passé de $25 \%$ à $47 \%$ en équilibrant la présence des juifs. Pourtant, il est difficile d'accepter l'argument de Havard selon lequel il fut obligé de changer son attitude face aux juifs du fait du climat antisémite très fort qui existait dans la ville et qui provoquait la méfiance des Grecs envers la Mission et son rôle. Son effort pour se présenter lui-même comme le réformateur de l'école et comme celui qui a rétabli la confiance des orthodoxes face à la présence française peut s'expliquer facilement si on pense au destinataire de son rapport. Cet effort de Havard pour présenter son activité comme une preuve de l'éloignement des juifs de l'école, était opportuniste à cause de l'antisémitisme qui dominait à l'époque la France vichyste.

Les changements apportés par Havard au programme des cours ont augmenté le nombre des écoliers tout en attirant, en même temps, du monde pour les cours destinés aux adultes. L'assemblée générale de la Mlf à Paris en 1937 a manifesté sa satisfaction pour le progrès du Lycée de Thessalonique sous la direction de Havard en soulignant que l'établissement, malgré les problèmes des dernières années, restait florissant. 
Pourtant, cette augmentation du nombre des élèves était plutôt factice et concernait l'organisation des cours quotidiens (du matin au soir sans interruption) pour les étudiants, les fonctionnaires du secteur public, les policiers, les militaires et les autres professionnels. En plus, pour obtenir une amélioration des résultats aux examens du bac français, Havard a doublé les heures d'enseignement du français au détriment de celles destinées à l'enseignement de la langue, de l'histoire et de la géographie de la Grèce. Grâce à l'action de Havard, une chaire de langue et de littérature françaises fut fondée en 1937 dans la section des langues étrangères de l'Université de Thessalonique. Il faut noter qu'en 1938 le Lycée a été reconnu par l'État grec comme une des institutions officielles de la France en Grèce bien que cet accord culturel entre les deux pays ne reconnaisse comme telles que l'École française d'Athènes et l'Institut français d'Athènes.

Le tableau suivant présente le nombre des élèves de la Mission, leur religion et les aides attribués par l'État français :

\begin{tabular}{|l|l|l|l|l|l|}
\hline Années & Élèves & Orthodoxes & Juifs & Autres & $\begin{array}{l}\text { Attribution française } \\
\text { (en FF) }\end{array}$ \\
\hline $1930-31$ & 519 & $26 \%$ & $71,2 \%$ & $2,8 \%$ & 1.011 .000 \\
\hline $1931-32$ & 459 & $23 \%$ & $74,7 \%$ & $2,3 \%$ & 871.000 \\
\hline $1932-33$ & 397 & $34,7 \%$ & $60,1 \%$ & $5,2 \%$ & 842.000 \\
\hline $1933-34$ & 424 & $34,8 \%$ & $58,54 \%$ & $6,66 \%$ & 678.000 \\
\hline $1934-35$ & 383 & $34,81 \%$ & $61 \%$ & $4,19 \%$ & 760.000 \\
\hline $1935-36$ & 345 & $37,33 \%$ & $59,3 \%$ & $3,37 \%$ & 648.000 \\
\hline $1936-37$ & 387 & $35,85 \%$ & $58,88 \%$ & $5,27 \%$ & 672.000 \\
\hline $1937-38$ & 459 & $46,79 \%$ & $47,32 \%$ & $5,89 \%$ & 788.000 \\
\hline $1938-39$ & 644 & $43,96 \%$ & $53,78 \%$ & $3,26 \%$ & 1.032 .000 \\
\hline $1939-40$ & 662 & $47,13 \%$ & $47,13 \%$ & $1,27 \%$ & 753.000 \\
\hline
\end{tabular}

16 À côté de l'activité de la Mission, il faut noter l'existence de deux associations qui se lièrent avec l'institution française. En 1912, un groupe d'anciens élèves de la Mission a fondé l' "Association des anciens et anciennes élèves de la Mission laïque de Salonique » sur une initiative du directeur de l'école, Jules Lecoq. Son but était la propagation des idées et de la langue françaises. Pour cette raison, elle a essayé d'organiser des colloques et des conférences, des bals, des cours postscolaires etc. malgré la situation de guerre de 1912 à 1918 et les problèmes posés par l'arrivée des émigrés en 1923 (AN, 20/4/1937). En 1930, une «Association franco-hellénique des anciens élèves des universités et des Grandes écoles de France " a été fondée à Thessalonique. La majorité des membres de cette association était composée par des 
anciens élèves de la Mission ou même des membres des deux associations. Le but essentiel de cette deuxième association était de renforcer les liens intellectuels et moraux entre les deux pays et particulièrement d'assurer la coopération intellectuelle entre les universités des deux pays.

L'impact de la guerre en matière de présence culturelle de la France à Thessalonique était bien réel. La ville située à la proximité des deux fronts de la guerre (frontières gréco-albanaise et gréco-yougoslave) a souffert des bombardements fréquents de l'aviation de l'Axe. Plusieurs bâtiments du quartier dit « des Français » ont été détruits, y compris l'église catholique de la ville. L'école des Filles de la Charité et l'hôpital français ont été transformés en hôpitaux militaires pour les besoins de l'armée grecque. L'école Jean Baptiste de la Salle a été transformée en caserne et les moines-enseignants furent obligés de déménager dans leur résidence d'été (AMAE, 18/6/1941).

Le lycée de la Mission laïque a été occupé par les troupes allemandes après leur invasion. Les Allemands ont saisi les bureaux des professeurs de l'école qui ont été forcés de partir pour Athènes. Seuls, le directeur de l'école, Havard, et un professeur sont restés sur place. Les nouveaux bâtiments du lycée ont abrité un hôpital militaire allemand et les cours ont été annulés sine die. La direction de l'école soulignait que la situation était plus que dramatique à cause de l'utilisation successive de l'école par l'armée grecque et l'armée allemande (AMAE, 1941). Les Allemands avaient détruit une partie des biens mobiliers de l'école - meubles, livres et documents administratifs. Ces problèmes ont été amplifiés quand la direction centrale de la Mission a décidé de suivre Charles de Gaulle en Angleterre. Par conséquent, la communication entre Thessalonique et les services centraux était plutôt difficile. Pour cette raison, au mois de septembre 1940, le service des œuvres françaises à l'étranger du ministère des Affaires étrangères a rebaptisé le lycée de la Mission en "Lycée Franco-hellénique de Thessalonique » et l'a placé sous son contrôle administratif (AMAE, 2/9/1940).

19 L'absence d'une grande institution française en fonction dans la ville et le contrôle administratif exclusif des Allemands ont permis aux effectifs allemands dans le domaine scolaire une progression marquante. Pendant l'année scolaire 1941-1942, les inscriptions à l'annexe de l'Académie de Munich ont touché 1200 personnes, c'est-àdire une augmentation de $50 \%$ sur l'année précédente. Le directeur de l'Académie, Otto Kielmeyer proposait l'adoption du modèle français pour l'expansion culturelle allemande dans la région et l'utilisation du statut d'occupant pour développer un plan d'action. Mais, au même moment, il notait l'absence d'aide financière de Berlin. D'après lui, l'Allemagne avait toutes les conditions pour attirer le peuple grec vers la culture allemande parce qu'elle n'avait pas de revendications territoriales comme l'Italie ou la Bulgarie. Après l'introduction de l'allemand dans les écoles grecques comme langue obligatoire, les inscriptions ont touché 2400 personnes (1942-1943). Cette évolution eut comme résultat que l'Académie de Thessalonique est devenue la plus grande d'Europe, juste derrière les Académies similaires de Paris et de Florence. Mais la vérité était que beaucoup de Grecs se sont inscrits à l'Académie pour des raisons opportunistes (par exemple, pour améliorer leurs rapports commerciaux avec ou grâce aux Allemands ou simplement pour bénéficier de la carte fournie par l'Académie, très utile lors des contrôles nazis) (Fleischer 1988 : 179-187).

Peu à peu les relations du consulat français de la ville avec les autorités allemandes sont devenues plus cordiales afin que les forces allemandes respectent les établissements français. Pourtant, cette amélioration des relations franco-allemandes eut comme 
résultat la perturbation des relations franco-grecques. Selon les rapports des services consulaires français, la majorité des Grecs n'approuvaient pas ce réchauffement des relations des Français avec les occupants. Certaines personnes percevaient d'ailleurs comme une provocation l'ancrage dans le port de Thessalonique d'un navire de guerre français. De plus, les autorités grecques, qui étaient au courant des problèmes administratifs de la Mission et des difficultés de communication que la direction de l'école avait avec la France, ont essayé - par voie juridique - de confisquer les biens du lycée sous prétexte que l'école devait de l'argent à l'État grec (AMAE, 23/10/1941). Cependant, certains responsables de l'administration de la ville voulaient saisir l'occasion afin de se débarrasser des Français en alléguant l'insatisfaction populaire quant aux rapports des «missionnaires » avec les Allemands. Une partie de la société locale d'avant-guerre - très proche de l'extrême droite - jugeait la Mission trop « judéophile ». Sans doute, l'expulsion des élèves juifs de l'école et l'extermination de la communauté israélite de la ville ont satisfait les extrémistes grecs qui, dans leur majorité, étaient plutôt favorables en ce qui concernait la présence des Allemands. Mais, en même temps, ils désiraient la purification culturelle de la société grecque des influences étrangères, notamment celles des Italiens et des Français qui avaient une forte tradition dans la région. L'hellénisation de l'enseignement pouvait passer à la deuxième phase : après l'homogénéisation des élèves, on pourrait passer à l'expulsion des «impérialistes de la culture». Cet esprit, qui confondait le nationalisme avec le patriotisme et la tradition, a trouvé un appui dans le comportement des Français face aux Allemands. Les nationalistes grecs qui adoptaient généralement les positions nazies, ont trouvé l'excuse nécessaire pour attirer la population: pourquoi fallait-il soutenir la présence du lycée français alors que les Français étaient amis des Allemands? En d'autres termes, les responsables de cette aventure judiciaire avec les Français se présentaient comme des patriotes qui voulaient punir la trahison française. Bien sûr, ils pourraient bénéficier des bâtiments modernes du lycée ! Et le paradoxe de cette histoire est qu'à la fin, les relations des Français avec les Allemands de cette ville ont sauvé les bâtiments du lycée d'une confiscation grecque!

21 Malgré l'avis du consul général français de la ville selon lequel les circonstances étaient en réalité positives pour une évolution de l'activité des écoles françaises, la vérité se présentait de fait beaucoup plus inquiétante. L'extermination des juifs par les nazis a privé les écoles françaises de la moitié de leur clientèle. Ce phénomène semblait encore plus grave à cause de la fermeture définitive des écoles de l'Alliance israélite universelle. L'optimisme du consul que les écoles françaises pourraient attirer les élèves grecs orthodoxes à partir du moment où un grand nombre d'écoles publiques restaient fermées ou dont le fonctionnement était problématique, était sans fondement (AMAE, 7/8/1941). Les écoles françaises connaissaient une série de problèmes qui ne permettaient pas l'optimisme ou de grands plans. Le manque de personnel, l'absence de ressources, les difficultés de la guerre et la méfiance de la population ne favorisaient ni un développement de l'activité culturelle ni une augmentation des inscriptions comme c'était le cas à Athènes. Le SOFE avait mis comme priorité la stabilisation et la continuité de l'activité culturelle de l'EFA, de l'IFA et des écoles des Cyclades en laissant Thessalonique au deuxième plan (AMAE, 19/9/1941). Bien sûr, le SOFE a essayé de préserver la présence culturelle française à Thessalonique mais les résultats étaient maigres. Ses demandes auprès du service d'armistice à Wiesbaden pour une évacuation immédiate de tous les bâtiments français de la ville par les forces allemandes n'eurent aucun résultat (AMAE, 17/9/1941). Peu à peu, en voyant les nazis accomplir le génocide 
des juifs, les responsables de la diplomatie françaises ont compris que la présence française dans la ville pourrait bien prendre fin même avant la fin de la guerre. Pour cette raison on a l'impression que Thessalonique a été laissée de côté par les Français pendant cette période.

\section{Conclusion}

$\mathrm{Au}$ lendemain de la guerre, la langue française est en déclin du fait de l'extermination de la population juive de la ville, traditionnellement francophile et francophone, et le lycée de la Mission laïque perd un grand nombre d'élèves. L'extermination de la majorité de la communauté israélite a privé le lycée de la Mission laïque d'un grand nombre d'élèves. De plus, les conflits de la guerre civile rendirent impossibles avant 1950 le fonctionnement régulier de l'école (AMAE, 31/10/1949). Les Français ont cherché à attirer les élèves qui dans le passé préféraient le lycée allemand de leur ville, lycée qui avait cessé ses activités avec la fin de la guerre (AMAE, 24/1/1946). Et en effet, le lycée de la Mission, qui a pu attirer l'ensemble des anciens élèves des écoles allemandes, italiennes et roumaines, verra ses effectifs augmenter jusqu'à 548 élèves, retrouvant ainsi au deuxième trimestre 1947, son niveau d'avant-guerre (Bulletin de MfF 1947: 6). Le lycée deviendra progressivement un centre de langue à l'image des instituts français des autres pays. Il essayera de répondre aux besoins d'une société salonicienne profondément transformée et touchée par les expériences de la période de la guerre mondiale et de la guerre civile qui suivit. Pendant toute la période 1950-1970 le lycée accueillait entre 800 et 1300 élèves et son centre culturel a développé une activité remarquable. Dans le domaine de l'enseignement, les annexes fondées au début des années cinquante à Kavala et à Drama n'ont pas réussi à survivre. De même, le fonctionnement, dans les années soixante, de deux nouvelles annexes à Serres et à Sidirokastron n'a pas duré longtemps. Pourtant, pour la fondation parisienne le rôle du lycée de Thessalonique restait très important, car en 1967 il représentait environ le 12\% de l'ensemble mondial des élèves de la Mission. En 1968, le lycée a pris le nom d' "Institut français de Thessalonique », un fait révélateur de la volonté de développer une activité culturelle plus élargie que l'enseignement traditionnel du français. Mais l'incendie, au mois de mars 1968, qui a détruit le bâtiment central de l'institution, a annulé une partie de la programmation pour la nouvelle décennie. Ce n'est qu'après 1971 que l'IFT reprit régulièrement ses activités.

BIBLIOGRAPHIE

\section{Sources primaires}

Archives du ministère des Affaires étrangères de Grèce (AYE), 1914-1916/B 33 (2), ministère des Affaires étrangères au ministère de l'Éducation, Athènes, 14 janvier 1914. 
AYE, 1914-1916/B 33 (2), ministère de l'Éducation au ministère des Affaires étrangères, Athènes, 16 juin 1914.

AYE, 1924/B 37, Gouvernement général de Thessalonique (Direction des Affaires Intérieures) au ministère des Affaires étrangères, Thessalonique, 8 décembre 1928.

Archives du ministère des Affaires étrangères (AMAE), Guerres 1939-1945, CEuvres 1940-1944, Enseignement, $n^{\circ} 42$, Compte-rendu de gestion du lycée de Salonique présenté par M. André Havard, Salonique, septembre 1940.

AMAE, Guerres 1939-1945, CEuvres 1940-1944, Enseignement, vol. 42, Service des œuvres françaises à l'étranger (SOFE) au Lycée de Salonique, $n^{\circ}$ 6, Vichy 2 septembre 1940.

AMAE, Guerre 1939-40, Vichy-Europe, vol. 401, Consulat de Salonique au Ministère, $\mathrm{n}^{\circ} 11$, Salonique 18 juin 1941.

AMAE, Guerres 1939-1945, Cuvres 1940-1944, Enseignement, vol. 42, Consulat de Salonique à l'Ambassade d'Athènes, $\mathrm{n}^{\circ}$ 115, Salonique 23 octobre 1941.

AMAE, Guerre 1939-40, Vichy-Europe, vol. 401, Consulat de Salonique au Ministère, $\mathrm{n}^{\circ} 18$, Salonique 7 août 1941.

AMAE, Guerre 1939-40, Vichy-Europe, vol. 401, MAE au ministère de la Défense, Vichy 19 septembre 1941

AMAE, Guerre 1939-40, Vichy-Europe, vol. 401, SOFE au Consulat de Salonique, $\mathrm{n}^{\circ} 111$, Vichy 17 septembre 1941.

AMAE, Relations Culturelles 1945-1947, Enseignement, 69, Consulat de Thessalonique à MAE, nº 2, Thessalonique, 24 janvier 1946.

AMAE, Relations Culturelles 1945-1947, Enseignement, 69, Consulat de Thessalonique à MAE, $\mathrm{n}^{\circ}$ 12, Thessalonique, 31 novembre 1946.

AMAE, Relations Culturelles 1945-1947, Enseignement, 69, Consulat de Thessalonique à MAE, $\mathrm{n}^{\circ}$ 51, Thessalonique 10 février 1947.

AMAE, Europe 1944-1960, Grèce, 148, Consulat de Thessalonique à MAE, nº 109-111, Thessalonique, 31 octobre 1949.

Archives Diplomatiques de Nantes (ADN), Athènes, série $\mathrm{A}, \mathrm{n}^{\circ} 424$, Consul général Wiet à l'ambassade française d'Athènes, $\mathrm{n}^{\circ}$ 44, Thessalonique 3 avril 1922.

$\mathrm{ADN}$, Athènes, série $\mathrm{B}, 119$, Association des anciens élèves de la Mission laïque de Salonique à SOFE, $\mathrm{n}^{\circ}$ 3, Thessalonique, 1939.

Archives Nationales (AN), 312 AP, dossier 12, Association des anciens et anciennes élèves de la Mission laïque de Salonique à Jean Zay, Salonique 20 avril 1937.

Archives de la Mlf, Rapport de $35^{\mathrm{e}}$ Assemblé générale, 1937, 10-11.

Archives de la Mlf, Rapport de 42e Assemblé générale, 9 juin 1949.

Bulletin de la Mlf, 39e Assemblée générale de la Mlf, 1946

Bulletin de la Mlf, 40 Assemblée générale de la Mlf, 1947.

Revue de l'enseignement du français hors de France 62 (juin-juillet 1926), 268-269.

Revue de l'enseignement du français hors de la France 84 (février-mars 1930), 71-74. 


\section{Sources secondaires}

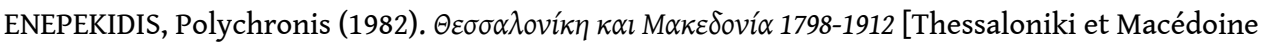
1798-1912]. Athènes : Estia.

DUMONT, Paul (1995). « La correspondance de Joseph Nehama avec l'Alliance israélite

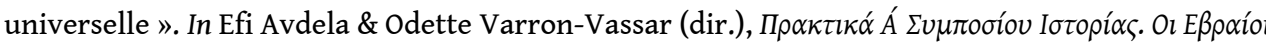

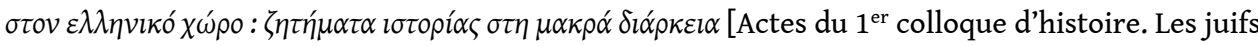
dans l'espace grec : questions d'histoire dans la longue durée]. Athènes : Gavriilidis, 131-150.

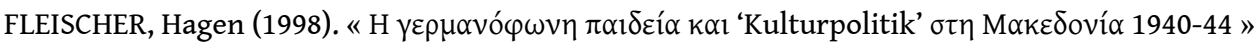
[L'éducation germanophone et la Kulturpolitik en Macédoine 1940-44]. In M $\alpha \kappa \varepsilon \delta o v i ́ \alpha \kappa \alpha l ~ \Theta \rho \alpha ́ \kappa \eta$

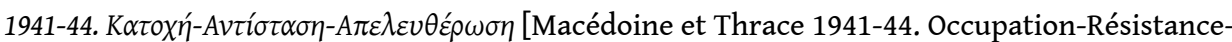
Libération]. Thessaloniki : Institut of Balkan Studies], 179-187.

GRUMEL-JACQUIGNON, François (2002). « Un exemple d'illusion géostratégique: la route de Salonique (1926-1933)». Relations Internationales, 109, 49-68.

HATZOPOULOS, Miltiade \& HAUTEFEUILLE, V. (1992). « Le journal intime de Léon Ray : un témoignage exceptionnel sur le service archéologique de l'armée d'Orient et sur la vie dans le camp retranché de Salonique ». In La France et la Grèce dans la Grande Guerre (actes du colloque tenu en novembre 1989 à Thessalonique). Thessalonique : Université de Thessalonique - Institut d'histoire des conflits contemporains, 191-200.

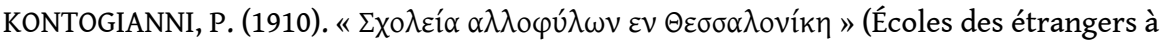

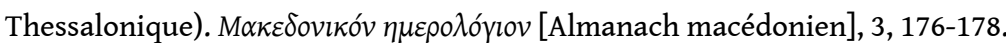

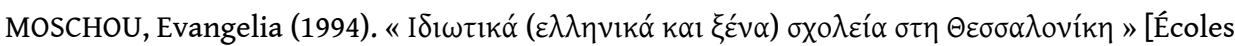

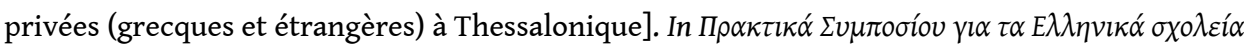

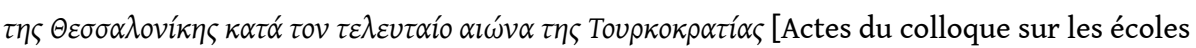
grecques de Thessalonique pendant le dernier siècle de l'occupation turque]. Thessalonique : Centre d'histoire de la ville, 267-275.

MOUTSOPOULOS, Nikolaos C. (1992). « Une ville entre deux siècles ». In Gilles Veistein (dir.). Salonique 1850-1918. La 'ville des juifs' et le réveil des Balkans. Paris : Autrement, 22-32.

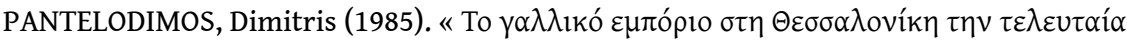

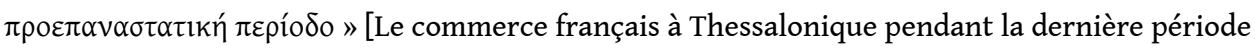

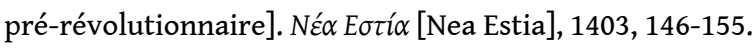

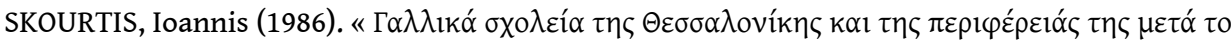

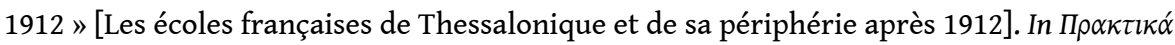

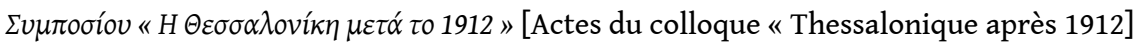

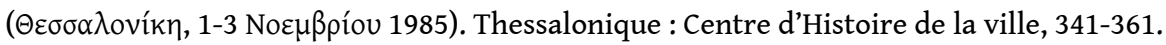

SKOURTIS, Yannis (1992). « L'Armée française d'Orient et ses travaux d'intérêt public en Grèce du Nord ». In La France et la Grèce dans la Grande Guerre (Actes du colloque tenu en novembre 1989 à Thessalonique). Thessalonique : Université de Thessalonique-Institut d'Histoire des conflits contemporains, 201-205.

THOBIE, Jacques (1977). Intérêts et impérialisme français dans l'empire ottoman (1895-1914). Paris : Publications de la Sorbonne-Imprimerie Nationale.

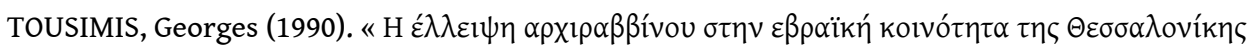

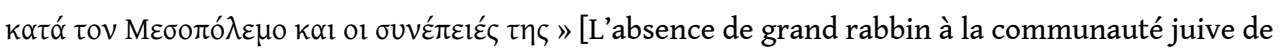

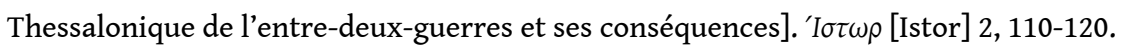




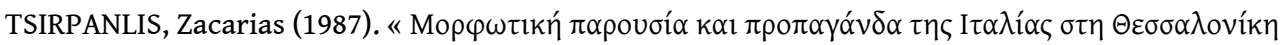

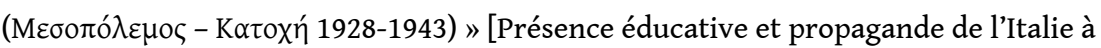
Thessalonique (Entre-deux-guerres - Occupation 1928-1943]. $\Delta \omega \delta \omega v \eta$ [Dodoni], 16/4.

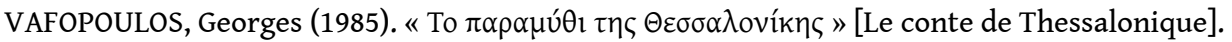

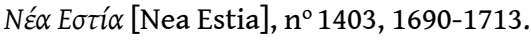

\section{NOTES}

1. Salonique fut la version occidentalisée ou française du nom turc de la ville Selanik. Après l'annexion de la ville en Grèce le nom officiel est Thessalonique ou Thessaloniki.

\section{RÉSUMÉS}

Le lycée de la Mission laïque française a joué depuis sa fondation en 1906 un rôle majeur dans la promotion de la francophonie et de la culture française dans la ville de Thessalonique. Situé dans une ville multiethnique et avec une tradition francophile, le lycée fut le berceau d'une activité linguistique, culturelle et spirituelle d'exception. Avec une clientèle notamment juive, la fondation se trouva également au milieu des antagonismes des communautés de la ville. Le paroxysme nationaliste, les obstacles posés par l'État grec et les propagandes étrangères concurrentes ont perturbé l'activité du lycée. Pendant les années 1930 et 1940 on assiste à la montée d'un antisémitisme remarquable au lycée. L'extermination de la communauté juive par les nazis et le déclin de la présence de la culture française après la guerre eurent pour résultat un changement dans les orientations du lycée. La fondation deviendra dans les années 1950 et 1960 un centre de langue à l'image des instituts linguistiques des autres pays et elle essayera de répondre aux besoins d'une société salonicienne profondément transformée et touchée par les expériences de la période de la guerre mondiale et de la guerre civile.

The Lycée de la Mission Laïque française since its founding in 1906 has played a major role in promoting the Francophonie and the French culture in the city of Thessaloniki. Situated in a multi-ethnic city with a Francophile tradition, the Lycée was the birthplace of a linguistic cultural and spiritual activity of exception. Particularly with a Jewish clientele, the foundation was in middle of the antagonisms between the communities of the city. The nationalist climate, the obstacles posed by the Greek state and the acceleration of the competition in the field of foreign propaganda disrupted the activity of the Lycée. The period of the 1930s and the years of the war are characterized by the birth and the evolution of an anti-Semitism in the middle of the Schools staff. The extermination of the Jewish community by the Nazis and the decline of the presence of French culture after the war had resulted the change of the directions of the School. The foundation will become in the 1950s and 1960s, a language center in the image of language institutes of the other countries and it will try to meet the needs of a local society profoundly different and affected by the experiences of the World War and the Greek civil war. 
INDEX

Mots-clés : Mission laïque française, francophonie, relations culturelles, Thessalonique, juifs, enseignement

Keywords : Mission laïque française, French langage, cultural relations, Thessaloniki, Jews, instruction

\section{AUTEUR}

\section{LAMPROS FLITOURIS}

Université de Ioannina 